\title{
On a Liouville-type equation with sign-changing weight
}

\author{
Bernhard Ruf* \\ Dipartimento de Matematica, Università degli Studi \\ Via Saldini 50, I-20133 Milano, Italy \\ ruf@mat.unimi.it \\ Pedro Ubilla ${ }^{\dagger}$ \\ Universidad de Santiago de Chile \\ Casilla 307, Correo 2, Santiago - Chile \\ pubilla@usach.cl \\ January 8, 2007
}

\begin{abstract}
In this paper we study the existence, nonexistence and multiplicity of non-negative solutions for the family of problems

$$
-\Delta u=\lambda\left(a(x) e^{u}+f(x, u)\right), \quad u \in H_{0}^{1}(\Omega)
$$

where $\Omega$ is a bounded domain in $\mathbb{R}^{2}$ and $\lambda>0$ is a parameter. The coefficient $\mathrm{a}(\mathrm{x})$ is allowed to change sign. The techniques used in the proofs are a combination of upper and lower solutions, the TrudingerMoser inequality and variational methods. Note that when $f(x, u)=0$ the equation is of Liouville type.
\end{abstract}

Keywords and phrases: Liouville equation, Gelfand equation, multiplicity, variational method, Trudinger-Moser inequality

AMS Subject Classification: 35J20 and 35J60

\section{Introduction}

In this paper we study equations of Liouville-type (also called Gelfand equations), which have the form

$$
\left\{\begin{array}{c}
-\Delta u=\lambda a(x) e^{u} \text { in } \Omega \subset \mathbb{R}^{2} \\
u=0 \text { on } \partial \Omega
\end{array} .\right.
$$

\footnotetext{
*Supported by PRIN (Italy)

${ }^{\dagger}$ Supported by FONDECYT 1040990.
} 
Here, $\Omega \subset \mathbb{R}^{2}$ is a bounded domain, $\lambda$ is a positive parameter, and $a(x)$ is a bounded coefficient. This and related equations have received much attention in recent years.

On the one hand, this is due to the wide range of applications of this equation: it is used in astrophysics [4] and combustion theory [11], it is related to the prescribed Gaussian curvature problem in Riemannian geometry [14], to the mean field limit of vortices in Euler flows [6], to Onsager's formulation in statistical mechanics [3], to the Keller-Siegel system of chemotaxis [20], to the Chern-Simon-Higgs gauge theory [5], [18], and it has many other physical applications.

On the other hand, equation (1.1) is mathematically appealing since it has an interesting solution structure: If one assumes that the coefficient $a(x)$ is positive (as is done in most cases studied in the literature), one may easily prove, upon multiplication of the equation by the first eigenfunction of the Laplacian and subsequent integration, that equation (1.1) has no solution for $\lambda$ large. On the other hand, for $\lambda>0$ close to zero, a positive solution exists, and it is a local minimizer for the corresponding energy functional. One easily verifies that this solution tends to zero as $\lambda \rightarrow 0$. A second "large" solution was first found in [19], see also [7]; it corresponds to a Mountain-Pass solution, and one shows that it blows up for $\lambda \rightarrow 0$.

Such a solution structure is in fact also present in higher dimensions, in problems with so-called "concave-convex" nonlinearities, see the pioneering paper of Ambrosetti-Brezis-Cerami [1]; a particular example of an equation with such a structure is

$$
\left\{\begin{array}{cl}
-\Delta u=\lambda u^{q}+u^{p} & \text { in } \Omega \\
u>0 & \text { in } \Omega \\
u=0 & \text { on } \partial \Omega,
\end{array}\right.
$$

where $0 \leq q<1<p$. This equation was extensively studied in [1], and it was in particular shown there that if $p \leq 2^{*}-1$, where $2^{*}=\frac{2 N}{N-2}, N \geq 3$, then there exists $0<\Lambda<\infty$ such that (1.2) has at least two solutions for $\lambda<\Lambda$, at least one solution for $\lambda=\Lambda$, and no solution for $\lambda>\Lambda$. More recently, in [8] and [9], more general nonlinearities were considered which include nonlinearities of the form $f(x, u)=\lambda a(x) u^{q}+b(x) u^{p}$, where the coefficients $a(x), b(x)$ are allowed to change sign.

We also refer to the paper of F. Mignot, F. Murat and J.P. Puel; in [16] they consider equation (1.1) in dimensions $3 \leq N<10$, and prove that there exists a $\lambda_{0}>0$ such that the equation has at least one solution for $0<\lambda<\lambda_{0}$, and no solution for $\lambda \geq \lambda_{0}$. Particular attention is given to the behavior of the solutions near the "turning point" $\lambda_{0}$. This problem was also considered in the paper [13] by D.D. Joseph and T.S. Lundgren, who considered the equation on $\Omega=B_{1}(0)$, the unit ball in $\mathbb{R}^{n}$, and studied in great detail the solution behavior for radial solutions; an interesting dependence of the solution structure on the dimension of the domain was found. 
Returning to two dimensions, we note that exponential growth is in some sense a critical growth; Brezis-Merle showed in [2] that all distributional solutions of equation (1.1) are bounded, and they give examples of (positive) coefficients $a(x)$ such that the equation

$$
-\Delta u=a(x) e^{u^{\alpha}} \text { in } \Omega, u=0 \text { on } \partial \Omega
$$

has unbounded distributional solutions for $\alpha>1$.

Interesting studies have recently been done concerning the blow-up of solutions of equation (1.1) when $\lambda \rightarrow 0$, with $a(x)>0$. Based on the results by Brezis-Merle it was shown in [15], [17] that the solutions necessarily blow up in "integer multiples" of $8 \pi$, more precisely, one has $\lim _{\lambda \rightarrow 0} \int_{\Omega} \lambda a(x) e^{u}=m 8 \pi$, for some $m \in \mathbb{N}$. In an interesting paper by M. del Pino, M. Kowalczik and M. Musso [10] it was recently shown that for any given $m \in \mathbb{N}$ such solutions indeed exist, provided that $\Omega$ is not simply connected.

In this paper we will consider the case that the coefficient $a(x)$ in equation (1.1) changes sign. In particular, we will assume that $a(x)$ is negative in a neighborhood of the boundary $\partial \Omega$, and is strictly positive on some open set in $\Omega$. We will show that then a similar solution behavior as the one mentioned above can be proved regarding non-negative solutions: we will show that there exist constants $0<\Lambda_{2} \leq \Lambda_{0}$ such that

- for $\lambda>\Lambda_{0}$ equation (1.1) has no non-negative solution

- for $0<\lambda<\Lambda_{2}$ equation (1.1) has at least two non-negative solutions

We emphasize that we look for non-negative solutions; we do not know whether there is non-existence for other type of solutions, for large $\lambda$. Also, we do not know if the non-negative solutions we find for small $\lambda$ are strictly positive, or if they could be equal to zero on some subset of $\Omega$.

As far as applications are concerned, we note that in the models in physics and biology it is reasonable to have negative reaction forces (i.e. $a(x)<0$ ) near the boundary of a domain. Also the search for non-negative solutions is well motivated, considering that $u(x)$ may be interpreted as a concentration or population density.

\section{Statement of the results}

Suppose that $\Omega \subset \mathbb{R}^{2}$ is a smooth bounded domain. We will consider the following more general form of equation (1.1):

$$
\left\{\begin{aligned}
-\Delta u=\lambda\left(a(x) e^{u}+f(x, u)\right) & \text { in } \Omega \\
u \geq 0, u \neq \equiv 0 & \text { in } \Omega \\
u=0 & \text { on } \partial \Omega
\end{aligned}\right.
$$


where $\lambda>0$ is a parameter, $a \in L^{\infty}(\Omega)$, and $f: \Omega \times \mathbb{R} \rightarrow \mathbb{R}$ is a Caratheodory function. Throughout this paper we suppose that $f(x, s)=0$ for all $s \leq 0$. We make the following assumptions on the coefficient $a(x)$ :

$\left(A_{-}\right)$There exists a positive constant $\delta$ such that

$$
\Omega_{\delta}:=\{x \in \Omega: d(x, \partial \Omega) \leq \delta\} \subseteq\{x \in \Omega: a(x) \leq 0\},
$$

and

$\left(A_{+}\right)$there exists a positive constant $\delta_{0}>0$ and a ball $B_{0} \subset \Omega$ such that

$$
a(x)>\delta_{0} \quad \text { for all } x \in B_{0} .
$$

On the function $f(x, s)$ we assume that it is a Caratheodory function satisfying the following hypotheses

$(G)$ There exist positive constants $d_{1}, d_{2}$ and $\sigma$ such that

$$
|f(x, s)| \leq d_{1}+d_{2} s^{\sigma}
$$

for a.e. $x \in \Omega$ and all $s \geq 0$.

$(P)$ There exist positive constants $c_{1}, c_{2}$ and $s_{0}$, and $q<2$ such that

$$
F(x, s)-f(x, s) \leq c_{1}+c_{2} s^{q},
$$

for a.e. $x \in \Omega$ and all $s \geq s_{0}$, where $F$ is given by $F(x, s)=\int_{0}^{s} f(x, t) d t$.

$\left(H_{1}\right)$ There exist positive constants $\delta_{1}, b_{1}, b_{2}$, and constants $q_{1}, q_{2}>1$ such that

$$
-b_{1} s^{q_{1}} \leq F(x, s) \leq b_{2} s^{q_{2}}
$$

for a.e. $x \in \Omega$ and all $0<s<\delta_{1}$.

The main results are the following

Theorem 2.1 (Multiplicity result). Under the assumptions $(G),\left(A_{-}\right),(P)$ and $\left(H_{1}\right)$ there exists a $\Lambda_{2}>0$ such that Problem (2.1) has at least two nonnegative solutions for all $0<\lambda<\Lambda_{2}$.

Theorem 2.2 (Non-existence result). Under the assumptions of Theorem 2.1 and the hypothesis

$\left(H_{2}\right)$ Let $B_{0}$ be as in hypothesis $\left(A_{+}\right)$, and assume that there exists $0 \leq m(x) \in$ $L^{r}\left(B_{0}\right) \backslash\{0\}$, for some $r>1$, such that

$$
a(x) e^{s}+f(x, s) \geq m(x) s \text { for all } x \in B_{0} \text { and } s \geq 0 .
$$

Then there exists a $\Lambda_{0}>0$ such that Problem (2.1) has no non-negative solutions for all $\lambda>\Lambda_{0}$. We note that $\Lambda_{0}$ may be chosen as $\lambda_{1}\left(m(x), \Omega_{1}\right)$, the first eigenvalue with weight $m$ on $\Omega_{1}$, where $\Omega_{1}$ is a ball with $\Omega_{1} \subset \subset B_{0}$. 


\section{The auxiliary problem}

We consider first the following auxiliary problems, with $k \in \mathbb{N}$

$$
\left\{\begin{array}{cl}
-\Delta u=\lambda\left(a(x) e^{k u^{-}} e^{u^{+}}+f(x, u)\right) & \text { in } \Omega \\
u>0 & \text { in } \Omega \\
u=0 & \text { on } \partial \Omega .
\end{array}\right.
$$

The associated energy functionals are given by

$$
J_{k}(u):=\frac{1}{2} \int_{\Omega}|\nabla u|^{2}-\int_{\Omega} \lambda\left(G_{k}(x, u)+F(x, u)\right), J_{k}: H_{0}^{1}(\Omega) \rightarrow \mathbb{R},
$$

where $G_{k}(x, s):=a(x)\left(e^{s}-1\right)$ if $s \geq 0$ and $G_{k}(x, s):=\frac{1}{k} a(x)\left(e^{k s}-1\right)$ if $s<0$.

The following lemma concerns the Palais-Smale condition for the functionals $J_{k}$ defined above

Lemma 3.1 Under the assumptions $(G),\left(A_{-}\right)$and $(P)$ the functionals $J_{k}$, $k \in \mathbb{N}$, satisfy the Palais-Smale condition.

Proof. Let $\left\{u_{n}\right\}$ be a $(\mathrm{PS})_{c}$ sequence, i.e.

$$
\begin{gathered}
c_{n}:=\frac{1}{2}\left\|u_{n}\right\|^{2}-\int_{\Omega} \lambda\left(G_{k}\left(x, u_{n}\right)+F\left(x, u_{n}\right)\right) \rightarrow c, \\
\left|\int_{\Omega} \nabla u_{n} \cdot \nabla \phi-\int_{\Omega} \lambda\left(a(x) e^{k u_{n}^{-}} e^{u_{n}^{+}}+f\left(x, u_{n}\right)\right) \phi\right| \leq \varepsilon_{n}\|\phi\|, \quad \forall \phi \in H_{0}^{1}(\Omega),
\end{gathered}
$$

From (3.2) and $\left(A_{-}\right)$we have

$$
\begin{gathered}
\frac{1}{2}\left\|u_{n}\right\|^{2}+\int_{\Omega_{\delta} \cap\left\{u_{n}>0\right\}} \lambda|a(x)| e^{u_{n}^{+}}+\int_{\Omega_{\delta} \cap\left\{u_{n}<0\right\}} \lambda \frac{1}{k}|a(x)| e^{k u_{n}^{-}}= \\
\int_{\Omega} \lambda F\left(x, u_{n}\right)+\int_{\Omega_{\delta}^{c} \cap\left\{u_{n}>0\right\}} \lambda a(x) e^{u_{n}^{+}}+\int_{\Omega_{\delta}^{c} \cap\left\{u_{n}<0\right\}} \lambda \frac{1}{k} a(x) e^{k u_{n}^{-}} \\
-\int_{\left\{u_{n}>0\right\}} \lambda a(x)-\int_{\left\{u_{n}<0\right\}} \lambda \frac{1}{k} a(x)+c_{n} .
\end{gathered}
$$

On the other hand, let $\phi \in C_{c}^{\infty}(\Omega)$ be such that $\phi=1$ in $\Omega_{\delta}^{c}$ and $0 \leq \phi \leq 1$ in $\Omega_{\delta}$. Hence, by using (3.3) we have

$$
\begin{aligned}
& \int_{\Omega_{\delta}^{c} \cap\left\{u_{n}>0\right\}} \lambda a(x) e^{u_{n}^{+}}+\int_{\Omega_{\delta}^{c} \cap\left\{u_{n}<0\right\}} \lambda \frac{1}{k} a(x) e^{k u_{n}^{-}} \leq \\
& \left\|u_{n}\right\|\|\phi\|+\int_{\Omega_{\delta} \cap\left\{u_{n}>0\right\}} \lambda|a(x)| e^{u_{n}^{+}}+\int_{\Omega_{\delta} \cap\left\{u_{n}<0\right\}} \lambda \frac{1}{k}|a(x)| e^{k u_{n}^{-}} \\
& \quad-\int_{\Omega} \lambda f\left(x, u_{n}\right) \phi+\epsilon_{n}\|\phi\| .
\end{aligned}
$$


And so, by combining the inequalities above we obtain

$$
\begin{aligned}
\frac{1}{2}\left\|u_{n}\right\|^{2} \leq & \int_{\Omega} \lambda\left(F\left(x, u_{n}\right)-f\left(x, u_{n}\right) \phi\right)+\left\|u_{n}\right\|\|\phi\| \\
& -\int_{\Omega} \lambda a(x)-\int_{\left\{u_{n}>0\right\}} \lambda \frac{1}{k} a(x)+\epsilon_{n}\|\phi\|+c_{n}
\end{aligned}
$$

On the other hand, from condition $(P)$ it is not difficult to show that there exist constants $C_{0}, C_{1}$ such that

$$
\int_{\Omega} \lambda\left(F\left(x, u_{n}\right)-f\left(x, u_{n}\right) \phi\right) \leq C_{1}\left\|u_{n}\right\|^{q}+C_{0}
$$

with $q<2$. Hence (3.5) and (3.6) allow us to conclude that the Palais-Smale sequence $\left\{u_{n}\right\}$ is bounded.

Thus, $\left\{u_{n}\right\}$ has a subsequence which converges weakly in $H_{0}^{1}(\Omega), u_{n}(x) \rightarrow$ $u(x)$ pointwise for a.e. $x \in \Omega$, and $u_{n} \rightarrow u$ in $L^{p}(\Omega)$, for every $1 \leq p<+\infty$. From this we obtain, by choosing $\varphi=u_{n}-u$ in (3.3), that

$$
\begin{gathered}
\left|\int_{\Omega} \nabla u_{n} \nabla\left(u_{n}-u\right) d x\right|=\mid \int_{\Omega} \lambda\left(a(x) e^{k u_{n}^{-}} e^{u_{n}^{+}}+f\left(x, u_{n}\right)\right)\left(u_{n}-u\right) d x \\
\leq \lambda\left\|a(x) e^{k u_{n}^{-}} e^{u_{n}^{+}}\right\|_{L^{2}}\left\|u_{n}-u\right\|_{L^{2}}+\left\|f\left(x, u_{n}\right)\right\|_{L^{2}}\left\|u_{n}-u\right\|_{L^{2}}
\end{gathered}
$$

Clearly, $\left\|f\left(x, u_{n}\right)\right\|_{L^{2}} \leq c$, and also

$$
\begin{aligned}
& \left\|a(x) e^{k u_{n}^{-}} e^{u_{n}^{+}}\right\|_{L^{2}}^{2} \leq|a|_{L^{\infty}}^{2} \int_{\Omega} e^{2 u_{n}^{+}} d x \leq c \int_{\Omega} e^{\frac{2 u_{n}^{+}}{\left\|u_{n}^{+}\right\|_{H_{0}^{1}}}\left\|u_{n}^{+}\right\|_{H_{0}^{1}}} d x \\
& \leq c \int_{\Omega} e^{\left|\frac{u_{n}^{+}}{\left\|u_{n}^{+}\right\|_{H_{0}^{1}}}\right|^{2}} e^{\left\|u_{n}^{+}\right\|_{H_{0}^{1}}^{2}} d x \leq c_{1}
\end{aligned}
$$

by the Trudinger-Moser inequality. Hence,

$$
\lim _{n \rightarrow \infty} \int_{\Omega}\left|\nabla u_{n}\right|^{2} d x=\lim _{n \rightarrow \infty} \int_{\Omega} \nabla u_{n} \nabla u d x=\int_{\Omega}|\nabla u|^{2} d x
$$

and thus $u_{n} \rightarrow u$ in $H_{0}^{1}(\Omega)$.

Next, we prove the existence of Mountain-Pass solutions for the auxiliary equations (3.1).

Lemma 3.2 Under the assumptions $(G),\left(A_{-}\right),\left(A_{+}\right),(P)$ and $\left(H_{1}\right)$ there exists a $\Lambda_{2}>0$ and $\alpha_{\lambda}>0$ such for all $\lambda \in\left(0, \Lambda_{2}\right)$ equation (3.1) has, for all $k \in \mathbb{N}$, a nontrivial solution $u_{k}$ which is mountain pass critical point of $J_{k}$ verifying $J_{k}\left(u_{k}\right)>\alpha_{\lambda}$, for all $k \in \mathbb{N}$. 
Proof. We note that conditions $(G)$ and $(P)$ imply that there exist $s_{1}>s_{0}$ and $\bar{\sigma}>q_{2}$ such that

$$
F(x, s) \leq f(x, s)+c_{1}+c_{2} s^{q} \leq c_{3}+c_{4} s^{\bar{\sigma}}
$$

for a.e. $x \in \Omega$ and all $s \geq s_{1}$. Thus from condition $\left(H_{1}\right)$ we have that there exists a large enough positive constant $b_{3}$ such that

$$
F(x, s) \leq b_{2} s^{q_{2}}+b_{3} s^{\bar{\sigma}}, \text { for all } s \geq 0 .
$$

Let $\lambda>0$, and set $\|u\|=\lambda^{\alpha}$ with $0<\alpha<\frac{1}{2}$. Thus from (3.7), Sobolev embeddings and the Trudinger-Moser inequality, for $\lambda$ small enough, we have that there exist constants $C_{1}, C_{2}, C_{3}>0$ such that

$$
\begin{aligned}
J_{k}(u) & =\frac{1}{2} \lambda^{2 \alpha}-\int_{\{u>0\}} \lambda\left[a(x)\left(e^{\frac{u}{\|u\|} \lambda^{\alpha}}-1\right)\right] d x \\
& -\int_{\{u<0\}} \lambda\left[\frac{1}{k} a(x)\left(e^{k u}-1\right)\right] d x-\int_{\Omega} \lambda F(x, u) d x \\
& \geq \frac{1}{2} \lambda^{2 \alpha}-\lambda C_{1}-C_{2} \lambda^{q_{2} \alpha+1}-C_{3} \lambda^{\bar{\sigma} \alpha+1}
\end{aligned}
$$

And so we can obtain a $\Lambda_{2}>0$ sufficiently small, such that for all $0<\lambda<\Lambda_{2}$ there is a $\alpha_{\lambda}>0$ such that

$$
J_{k}(u) \geq \alpha_{\lambda}, \text { for }\|u\|=\lambda^{\alpha}, \text { for all } k \in \mathbb{N} .
$$

Now let $\phi \in C_{c}^{\infty}(\Omega)$ be such that $\operatorname{supp} \phi \subset B_{0}$. Based on Lemmas 3.1 and 3.2 above and the classical Mountain Pass Theorem, to obtain a nontrivial solution it is sufficient to show that

$$
\lim _{t \rightarrow+\infty} J_{k}(t \phi)=-\infty
$$

Indeed, there exist a natural number $m>\max \{2, \sigma+1\}$ and a constant $c_{m}$ such that $e^{u}-1 \geq c_{m} u^{m}$ for $u \geq 0$. Thus from (3.7), $\left(A_{+}\right)$and $(G)$ we have

$$
J_{k}(t \phi) \leq \frac{1}{2} t^{2} \int_{B_{0}}|\nabla \phi|^{2}-\lambda c_{m} t^{m} \delta_{0} \int_{B_{0}} \phi^{m}+\lambda D_{1} t^{\sigma+1} \int_{B_{0}} \phi^{\sigma+1}+D_{2}
$$

where $D_{1}, D_{2}$ are constants. Therefore (3.9) holds and so there exists a solution $u_{k}$ verifying $J_{k}\left(u_{k}\right) \geq \alpha_{\lambda}$.

In the next Lemma we prove the existence of non-negative solutions which are local minima. 
Lemma 3.3 Under the assumptions $(G),\left(A_{-}\right),(P)$ and $\left(H_{1}\right)$ there exist $\lambda_{0}>0$ and $c_{\lambda}>0$ such for all $\lambda \in\left(0, \lambda_{0}\right)$ the equation (3.1) has a nontrivial solution $v_{k}$ which is local minimum of $J_{k}$ verifying $J_{k}\left(v_{k}\right)<-c_{\lambda}$, for all $k \in \mathbb{N}$.

Proof. Let $\phi \in C_{c}^{\infty}\left(B_{0}\right)$ as above. Using that $e^{t \phi}-1 \geq t \phi$, we obtain, for $t>0$ small enough

$$
J_{k}(t \phi) \leq \frac{1}{2} t^{2} \int_{B_{0}}|\nabla \phi|^{2}-\lambda t \int_{B_{0}} a(x) \phi+b_{1} \lambda t^{q_{1}} \int_{B_{0}}|\phi|^{q_{1}}
$$

Thus for every $\lambda>0$ there exists $t, c_{\lambda}>0$ such that

$$
\|t \phi\|<\lambda^{\alpha} \text { and } J_{k}(t \phi)<-c_{\lambda}
$$

And so, by using (3.8) and (3.11) there is a $\Lambda_{0}>0$ for all $\lambda \in\left(0, \Lambda_{0}\right)$ there exists a critical point $v_{k}$, such that $\left\|v_{k}\right\|<\lambda^{\alpha}$ is a local minimum with $J_{k}\left(v_{k}\right)<-c_{\lambda}$.

\section{Proof of the multiplicity result}

We now prove the main result Theorem 2.1. From Lemma 3.2 there exists a $\Lambda_{2}>0$ and $\alpha_{\lambda}>0$ such for all $\lambda \in\left(0, \Lambda_{2}\right)$ equation (3.1) has a weak nontrivial solution $u_{k}$ with $J_{k}\left(u_{k}\right)>\alpha_{\lambda}$, that is, for all $\varphi \in H_{0}^{1}(\Omega)$ we have

$$
\int_{\Omega} \nabla u_{k} \nabla \varphi=\lambda \int_{\Omega}\left(a(x) e^{k u_{k}^{-}+u_{k}^{+}}+f\left(x, u_{k}\right)\right) \varphi
$$

By using the same argument as in Lemma 3.2 we have that there is a constant $\tilde{c}$ such that $\left\|u_{k}\right\| \leq \tilde{c}$ for all $k \in \mathbb{N}$, and then the sequence $\left\{u_{k}\right\}$ converges weakly to some function $\bar{u}$ in $H_{0}^{1}(\Omega)$. It follows by the Trudinger-Moser inequality that

$$
\int_{\Omega}\left|e^{u_{k}^{+}}\right|^{2} d x=\int_{\Omega} e^{2 \frac{u_{k}^{+}}{\left\|u_{k}^{+}\right\|}\left\|u_{k}^{+}\right\|} \leq \int_{\Omega} e^{\left(\frac{u_{k}^{+}}{\left\|u_{k}^{+}\right\|}\right)^{2}+\left\|u_{k}^{+}\right\|^{2}} \leq c_{T M} e^{\left\|u_{k}^{+}\right\|^{2}} \leq c_{T M} c .
$$

By assumption $(G)$ we have that the sequence $\left\{\int_{\Omega}\left|f\left(x, u_{k}\right)\right|^{2} d x\right\}$ is bounded, and so the sequence $\left\{a(x) e^{k u_{k}^{-}+u_{k}^{+}}+f\left(x, u_{k}\right)\right\}$ is bounded in $L^{2}(\Omega)$. Thus by $L^{2}$-regularity we have that $\left\|u_{k}\right\|_{W^{2,2}} \leq c$. This implies that $\left\|u_{k}\right\|_{C^{0, \alpha}} \leq c$, $0<\alpha \leq 1$, and hence, for a subsequence, $u_{k} \rightarrow \bar{u}$ in $C^{0}$. We now distinguish two cases:

- if $x \in \Omega$ is such that $\bar{u}(x)<0$, then

$$
a(x) e^{k u_{k}^{-}(x)+u_{k}^{+}(x)} \rightarrow 0 \text { and } f\left(x, u_{k}(x)\right) u_{k}^{-}(x) \rightarrow 0
$$

- if $x \in \Omega$ such that $\bar{u}(x) \geq 0$, then

$$
\left(a(x) e^{k u_{k}^{-}(x)+u_{k}^{+}(x)}+f\left(x, u_{k}(x)\right) u_{k}^{-}(x) \rightarrow 0\right.
$$


Thus, by using the classical dominated convergence theorem, we can conclude that

$$
\int_{\Omega}\left(a(x) e^{k u_{k}^{-}+u_{k}^{+}}+f\left(x, u_{k}\right)\right) u_{k}^{-} \rightarrow 0 .
$$

Now, by passing to the limit $k \rightarrow \infty$ in (4.1), with $\varphi=u_{k}^{-}$, we obtain $\bar{u}^{-}=0$. Finally, taking the limit $k \rightarrow \infty$ in (4.1) we get

$$
\int_{\Omega} \nabla \bar{u} \nabla \varphi=\lambda \int_{\Omega}\left(a(x) \chi_{[\bar{u} \geq 0]} e^{\bar{u}^{+}}+f(x, \bar{u})\right) \varphi=\lambda \int_{\Omega}\left(a(x) e^{\bar{u}}+f(x, \bar{u})\right) \varphi
$$

since $\bar{u} \geq 0$. Thus, $\bar{u}$ is a non-negative solution of (2.1) with $J_{\lambda}(\bar{u}) \geq \alpha_{\lambda}$, where

$$
J_{\lambda}(u):=\frac{1}{2} \int_{\Omega}|\nabla u|^{2}-\int_{\Omega} \lambda(G(x, u)+F(x, u)),
$$

and $G(x, s):=a(x)\left(e^{s}-1\right), s \in \mathbb{R}$. Proceeding in a similar way, via Lemma 3.3, allows us to show the existence of second non-negative solution $\bar{v}$ of Problem (2.1) with $J_{\lambda}(\bar{v}) \leq-c_{\lambda}$.

Since the energy levels of the solutions $\bar{u}$ and $\bar{v}$ are different, we have found two distinct non-trivial solutions of equation (2.1).

\section{Proof of the non-existence result}

Let $g(x, s)=a(x) e^{s}+f(x, s)$ and consider the following eigenvalue problem

$$
\left\{\begin{aligned}
-\Delta w & =\lambda m(x) w & & \text { in } \Omega_{1} \\
w & =0 & & \text { on } \partial \Omega_{1} .
\end{aligned}\right.
$$

where $\Omega_{1}$ is a ball verifying $\Omega_{1} \subset \subset B_{0}$ and $m(x) \neq 0$ in $\Omega_{1}$. We will show that Problem (2.1) has no solutions for all $\lambda>\lambda_{1}(m(x))$, where $\lambda_{1}(m(x))$ is the first eigenvalue of (5.1). In fact, suppose the contrary, this is, Problem (2.1) has a solution for some $\hat{\lambda}>\lambda_{1}(m(x))$. So we may choose $\mu<\hat{\lambda}$ and $\mu \in\left(\lambda_{1}(m(x)), \lambda_{2}(m(x))\right)$. Let $\phi \in C_{c}^{\infty}\left(\Omega_{1}\right)$ and $\hat{u}$ the solution of (2.1) for that $\hat{\lambda}$, which can be assumed $C^{1}$. Indeed, since $\hat{u} \in H_{0}^{1}(\Omega)$ it follows by the Trudinger-Moser inequality and the assumptions on $a(x)$ and $f(x, s)$ that the righthand side of $(2.1)$ is in $L^{p}(\Omega)$, for any $p \geq 1$. Hence $\hat{u} \in W^{2, p}(\Omega)$, for any $p \geq 1$, and so $\hat{u} \in C^{1}(\Omega)$ by the classical Sobolev embedding. From $\left(H_{2}\right)$ we have

$$
\int_{\Omega_{1}} \nabla \hat{u} \nabla \phi=\int_{\Omega_{1}} \hat{\lambda}\left(a(x) e^{\hat{u}}+f(x, \hat{u})\right) \phi \geq \mu \int_{\Omega_{1}} m(x) \hat{u} \phi
$$

Thus the function $\hat{u}$ is a weak super-solution of Problem (5.1) for $\lambda=\mu$. But we may easily show that if $\phi_{1}$ is the first eigenfunction of (5.1) then for all $t \geq 0$ we have that $t \phi_{1}$ is a sub-solution of Problem (5.1) for $\lambda=\mu$. On the other hand, there exists a constant $b_{0}>0$ such that

$$
\hat{u}(x) \geq b_{0} \text { for all } x \in \bar{\Omega}_{1} .
$$


In fact, from hypotheses $\left(A_{+}\right)$and $\left(H_{2}\right)$ we have

$$
-\Delta \hat{u}=\hat{\lambda}\left(a(x) e^{\hat{u}}+f(x, \hat{u})\right) \geq 0, \text { for all } x \in B_{0} .
$$

The strong maximum principle can thus be applied (cf. e.g. Theorem 8.19 in [12]), which yields $\hat{u}(x)>0$ for all $x \in B_{0}$, and so we have (5.2). Finally by taking $t$ small enough we have that the sub-solution $t \phi_{1}$ satisfies $t \phi_{1} \leq \hat{u}$. Thus we obtain a solution of (5.1) for $\lambda=\mu$ with $\mu \in\left(\lambda_{1}(m(x)), \lambda_{2}(m(x))\right)$. This is clearly a contradiction because equation (5.1) has only the trivial solution for such $\mu$.

\section{References}

[1] A. Ambrosetti, H. Brezis and G. Cerami, Combined effects of concave and convex nonlinearities in some elliptic problems, J. Funct. Anal., 122 (1994), 519-543.

[2] H. Brezis, F. Merle, Uniform estimates and blow-up behavior for solutions of $-\Delta u=V(x) e^{u}$ in two dimensions, Comm. PDE 16 (1991), 1223-1253.

[3] E. Caglioti, P.L. Lions, C. Marchioro, M. Pulvirenti, A special class of stationary flows for two-dimensional Euler equations: a statistical mechanics description, I \& II, Comm. Math. Phys. 143 (1992), 501$525 \& 174$ (1995), 229-260

[4] S. Chandrasekhar, An introduction to the study of stellar structure, Dover, New York 1957

[5] D. Chae, O. Imanuvilov, The existence of non-topological multivortex solutions in the relativistic self-dual Chern-Simons theory, Comm. Math. Phys. 215 (2000), 119-142

[6] S. Chanillo, M. Kiessling, Rotational symmetry of solutions of some nonlinear problems in statistical mechanics and in geometry, Comm. Math. Phys. 160 (1994), 217-238

[7] M. Crandall, P.H. Rabinowitz, Some continuation and variational methods for positive solutions of nonlinear elliptic eigenvalue problems, Arch. Rational Mech. Anal. 58 (1975), 207-218

[8] D. de Figueiredo, J.-P. Gossez and P. Ubilla, Local superlinearity and sublinearity for indefinite semilinear elliptic problems, J. Funct. Anal., 199 (2003), 452-467.

[9] D. de Figueiredo, J.-P. Gossez and P. Ubilla, Multiplicity results for a family of semilinear elliptic problems under local superlinearity and sublinearity, J. Eur. Math. Soc., 8 (2006), no. 2, 269-286. 
[10] M. Del Pino, M. Kowalczyk and M. Musso, Singular limits in Liouvilletype equations, Calc. Var. Partial Differential Equations 24 (2005), 47-81.

[11] I.M. Gelfand, Some problems in the theory of quasilinear equations. Amer. Math. Soc. Transl. 29 (1963), 295-381

[12] D. Gilbarg, N.S. Trudinger, Elliptic partial differential equations of second order, Springer-Verlarg (1983).

[13] D.D. Joseph, T.S. Lundgren, Quasilinear problems driven by positive sources, Arch. Rat. Mech. Anal. 49 (1973), 241-269.

[14] J. Kazdan, F. Warner, Existence and conformal deformation of metrics with prescribed Gaussian and scalar curvatures, Ann. Math. 101 (1975), 317-331

[15] Y.-Y. Li, I. Shafrir, Blow-up analysis for solutions of $-\Delta u=V e^{u}$ in dimension two, Indiana Univ. Math. J. 43 (1994), 1255-1270

[16] F. Mignot, F. Murat and J.P. Puel, Variation d'un point de retournement par rapport au domaine, Comm. P.D.E. 4 (1979). 183-215.

[17] K. Nagasaki, T. Suzuki, Asymptotic analysis for two-dimensional elliptic eigenvalue problems with exponentially dominated nonlinearities, Asymptotic Anal. 3 (1990), 173-188

[18] M. Struwe, G. Tarantello, On multivortex solutions in Chern-Simons gauge theory, Boll. Unione Mat. Ital. Sez. B Artic. Ric. Mat. 8 (1998), 109-121

[19] V.H. Weston, On the asymptotic solution of a partial differential equation with an exponential nonlinearity, SIAM J. Math. Anal. 9 (1978), 10301053

[20] T. Senba, T. Suzuki, Some structures of the solutions set from stationary system of chemotaxis, Adv. Math. Sci. Appl. 10 (2000), 191-224. 